\title{
Critical region within $22 q 11.2$ linked to higher rate of autism spectrum disorder
}

\author{
Caitlin C. Clements ${ }^{1,2^{*}+}$, Tara L. Wenger ${ }^{1,3+}$, Alisa R. Zoltowski ${ }^{1,4}$, Jennifer R. Bertollo ${ }^{1}$, Judith S. Miller ${ }^{1,5}$, \\ Ashley B. de Marchena ${ }^{1,6}$, Lauren M. Mitteer ${ }^{7}$, John C. Carey ${ }^{8}$, Benjamin E. Yerys ${ }^{1,5}$, Elaine H. Zackai', \\ Beverly S. Emanuel ${ }^{7}$, Donna M. McDonald-McGinn ${ }^{7}$ and Robert T. Schultz ${ }^{1,5,7}$
}

\begin{abstract}
Background: Previous studies have reported no clear critical region for medical comorbidities in children with deletions or duplications of 22q11.2. The purpose of this study was to evaluate whether individuals with small nested deletions or duplications of the LCR-A to B region of 22q11.2 show an elevated rate of autism spectrum disorder (ASD) compared to individuals with deletions or duplications that do not include this region.
\end{abstract}

Methods: We recruited 46 patients with nested deletions $(n=33)$ or duplications ( $n=13$ ) of 22q11.2, including LCR-A to $B\left(n_{\text {del }}=11\right)$, LCR-A to $C\left(n_{\text {del }}=4\right)$, LCR-B to $D\left(n_{\text {del }}=14 ; n_{\text {dup }}=8\right)$, LCR-C to $D\left(n_{\text {del }}=4 ; n_{\text {dup }}=2\right)$, and smaller nested regions $(n=3)$. Parent questionnaire, record review, and, for a subset, in-person evaluation were used for ASD diagnostic classification. Rates of ASD in individuals with involvement of LCR-B to LCR-D were compared with Fisher's exact test to LCR-A to LCR-B for deletions, and to a previously published sample of LCR-A to LCR-D for duplications. The rates of medical comorbidities and psychiatric diagnoses were determined from questionnaires and chart review. We also report group mean differences on psychiatric questionnaires.

Results: Individuals with deletions involving LCR-A to B showed a 39-44\% rate of ASD compared to 0\% in individuals whose deletions did not involve LCR-A to B. We observed similar rates of medical comorbidities in individuals with involvement of LCR-A to $B$ and LCR-B to $D$ for both duplications and deletions, consistent with prior studies.

Conclusions: Children with nested deletions of 22q11.2 may be at greater risk for autism spectrum disorder if the region includes LCR-A to LCR-B. Replication is needed.

Keywords: 22q11.2 deletion syndrome, 22q11.2 duplication syndrome, Autism spectrum disorder, RANBP1, Screening, Atypical, Nested, Syndromic autism, Prosopagnosia, Face processing

\section{Background}

22q11.2 deletion syndrome (22q11.2DS), also known as DiGeorge or velocardiofacial syndrome, is the most common microdeletion syndrome in humans. The $3 \mathrm{Mb}$ region most frequently affected in 22q11.2DS can also be duplicated, resulting in 22q11.2 duplication syndrome (22q11.2DupS) [1]. Previous studies suggested a prevalence of 1 in 4000 live births for 22q11.2DS, but a recent study of consecutive pregnancies found an incidence of 1

\footnotetext{
* Correspondence: Clements@sas.upenn.edu

${ }^{\dagger}$ Equal contributors

${ }^{1}$ Center for Autism Research, The Children's Hospital of Philadelphia, 2716 South Street, Philadelphia, PA 19104, USA

2Department of Psychology, University of Pennsylvania, 3720 Walnut Street, Philadelphia, PA 19104, USA

Full list of author information is available at the end of the article
}

in 992 live births--similar to that of trisomy 21 [2]. The incidence of 22q11.2DupS was found to be 1 in 850 [2].

The 22q11.2 region includes low copy repeats (LCRs or segmental duplication blocks) interspersed throughout the region that frequently result in "breakpoints" for a duplication or deletion. The most commonly duplicated or deleted region spans LCR-A to LCR-D (hereafter--"classic" deletion/duplication). However, smaller nested deletions occur in $15 \%$ of affected individuals and usually stretch from only LCR-A to LCR-B, or from LCR-B to LCR-D, but can also span LCR-A to LCR-C or LCR-C to LCR-D [3]. In other cases, deletions include the area upstream of LCR-A or extend past LCR-D (see Fig. 1). The diagnoses of 22q11.2DS or 22q11.2DupS can refer to patients with either a classic or nested deletion/duplication. 


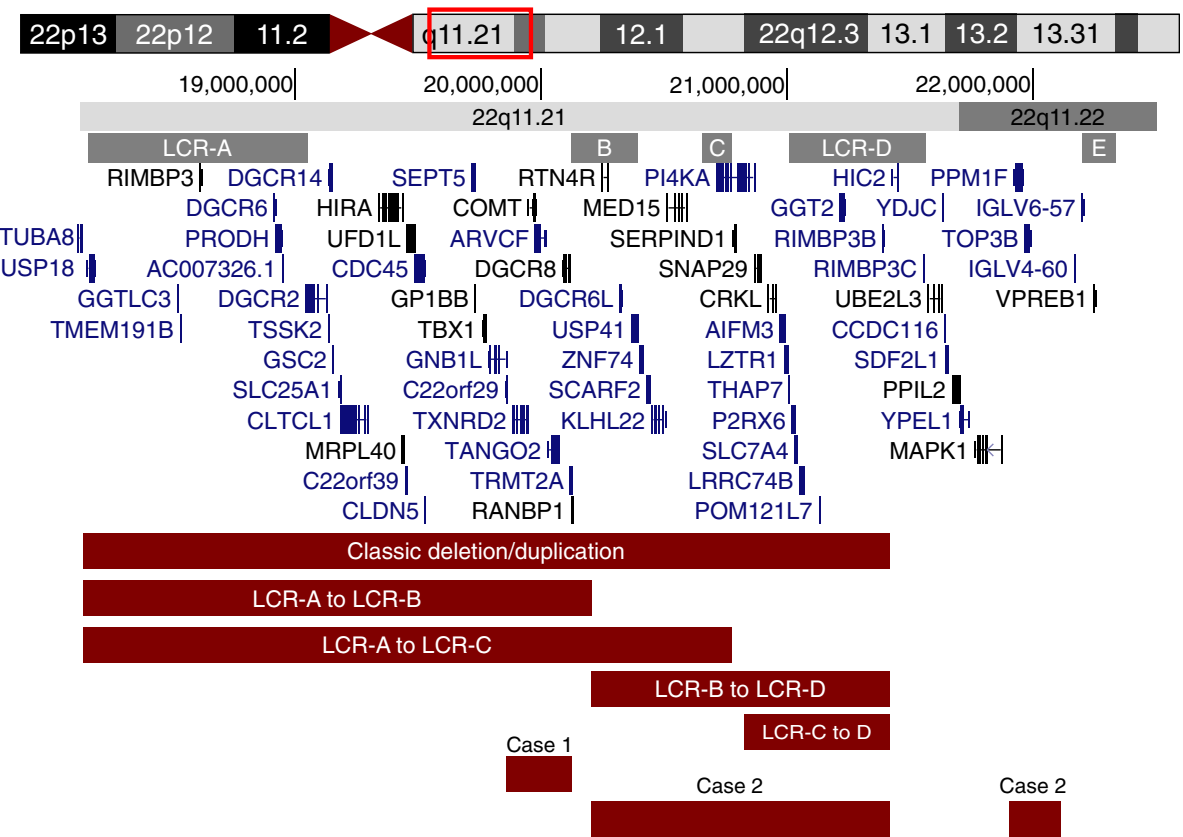

Fig. 1 22q11.2 diagram. Genes and low-copy repeat ("LCR") regions in the 22q11.2 region. Red bars depict deletions or duplications of participants. From GENCODE v24 genes in UCSC genome browser, December 2013 Assembly (genome.ucsc.edu)

The phenotypes of 22q11.2DS and 22q11.2DupS overlap with one another and show significant individual differences $[4,5]$. The syndromes can affect almost any organ system, and individuals can present with diverse constellations of medical issues and structural malformations, as well as a wide range of severity. Common medical comorbidities include congenital heart disease, hypocalcemia, renal abnormalities, immune deficiencies, and neuropsychiatric differences [1]. There is a recognizable facial gestalt in 22q11.2DS, but no recognizable gestalt has been identified in 22q11.2DupS. The rate of medical problems is much lower in 22q11.2DupS [6].

The 22q11.2 region has also been associated with elevated rates of autism spectrum disorder (from now on referred to as "ASD"), attention deficit/hyperactivity disorder (ADHD), and most notably, schizophrenia. A recent large study of 22q11.2DS reported psychosis in $41 \%$ of adults and ADHD in $37 \%$ of children [7], although a psychiatric registry-based study found lower rates [8]. Interestingly, there are no reported individuals with 22q11.2DupS with schizophrenia, and one group even suggested that it may be protective for schizophrenia [9]. In contrast, an elevated risk of ASD is found in both 22q11.2DS and 22q11.2DupS. As many as $50 \%$ of individuals with $22 \mathrm{q} 11.2 \mathrm{DS}$ and $38 \%$ with $22 \mathrm{q} 11.2 \mathrm{DupS}$ have received community diagnoses of autism spectrum disorder; however, fewer meet strict diagnostic criteria in research settings with reported rates of $0-18 \%$ in 22q11.2DS [10-12] and 14-25\% in 22q11.2DupS [6].
Despite significant heterogeneity in the 22q11.2 phenotype [13], little is known about critical regions that may confer risk for any specific part of the phenotype beyond schizophrenia, cleft palate, and cardiac anomalies. Prior reports point to TBX1, CRKL, and MAPK1 as contributors to the cardiac [14-18] and cleft palate phenotypes [19] in 22q11.2DS. Other research linked schizophrenia risk in 22q11.2DS to hyperprolinemia associated with lowered expression of proline dehydrogenase $(P R O D H)[20,21]$. Some studies reported an association between schizophrenia risk in 22q11.2DS and the lower activity Met allele of catechol-Omethyltransferase (COMT) [20, 22, 23], but larger cohort studies found no evidence [24-26] (for review, see [27]). These risk genes span the 22q11.2 region, with COMT, $P R O D H$, and TBX1 lying between LCR-A and LCR-B, while CRKL lies between LCR-C and LCR-D, and MAPK1 lies between LCR-D and LCR-E.

Recent research identified two genes as potential mediators of the ASD risk in 22q11.2DS. Radoeva et al. reported that in a sample of 87 individuals with 22q11.2DS, individuals with ASD were more likely to carry both the low-activity alleles of COMT and PRODH (leading to high levels of proline) than individuals without ASD [28]. Neither gene individually showed a significant direct relationship with ASD, although the pattern trended in that direction. Hidding et al. further demonstrated a quantitative relationship between ASD symptom severity and the combination of COMT-Met genotype and high proline levels in 45 individuals with 
22q11.2DS with and without ASD [29]. Both results suggest that the interaction between COMT and $P R O D H$, which lie in the LCR-A to B region, may increase ASD risk in individuals with 22q11.2DS.

The purpose of the present study was to leverage a novel study design to determine whether risk for autism can be narrowed to the LCR-A to LCR-B region within $22 \mathrm{q} 11.2$. Owing to the rarity of these nested structural variants, this is the first study to our knowledge that attempts to collect and phenotype large enough samples to test this hypothesis. We hypothesized that individuals harboring deleted LCR-A to LCR-B would show higher rates of ASD [30]; in addition to this region harboring $C O M T$ and $P R O D H$, it also contains RANBPI, a gene involved in the metabotropic glutamate receptor (mGluR) gene network that we previously hypothesized could play a role in ASD in 22q11.2DS/DupS [30]. In addition, we describe two case studies (one from our cohort and one from the literature) with much smaller, atypical duplications within the LCR-A to B region to gain hints as to the role of specific genes.

\section{Methods}

\section{Participants}

Participants with nested 22q11.2 duplications or deletions

Participants included 43 individuals with a nested duplication $(n=13)$ or deletion $(n=30)$ of $22 \mathrm{q} 11.2$ that lay entirely within LCR-A to LCR-D but was not completely inclusive of LCR-A to LCR-D (see Table 1). The only exception to this was one participant who carried a duplication of LCR-B to LCR-D and also a very small duplication between LCR-E and LCR-F. Participants were recruited from a specialty clinic at The Children's Hospital of Philadelphia (CHOP) or were referred from a similar specialty clinic at another institution. The CHOP " $22 \mathrm{q}$ and You" clinic represents the largest single-site 22q11.2 clinic in the world and maintains a large catchment area across the eastern US, with patients concentrated within a few hundred mile radius of CHOP. The sample includes probands who came to clinical attention, as well as their affected siblings ( $n=2$ with duplication and $n=3$ with deletion) and parents ( $n=2$ with duplication and $n=2$ with deletion) whose 22q11.2DS or 22q11.2DupS was identified after the proband's diagnostic process. The duplication or deletion was confirmed using single nucleotide polymorphism (SNP) microarray or Multiplex Ligation Probe Amplification (MLPA).

Samples whose copy number variations (CNVs) were tested by MLPA were examined using the SALSA P250 DiGeorge diagnostic probe kit (MRC-Holland, Amsterdam, The Netherlands). Commercially available software, Gene Marker from SoftGenetics (State College, PA), was used to analyze the data. Gene Marker has developed a completely integrated application for MLPA analysis with integrated functions specific for the analysis of data derived from MLPA reactions. Samples whose CNVs were identified by SNP array were analyzed using the Affymetrix SNP Array 6.0 platform following the manufacturer's instructions (Affymetrix, Santa Clara, CA, USA). Quality control values were calculated in Affymetrix Genotyping Console (Affymetrix), and any samples with contrast QC greater than 0.4 or mean absolute pairwise difference (MAPD) greater than 0.35 were excluded from further analysis. The B allele frequency and $\log \mathrm{R}$ ratio plots were visualized using the Affymetrix Chromosome Analysis Suite to support CNV calls.

Table 1 Descriptive characteristics of all participants in study

\begin{tabular}{|c|c|c|c|c|c|}
\hline Region & Number & $\%$ de novo ${ }^{a}$ & Age mean (sd) & Age range (years) & $\%$ male \\
\hline Total & 46 & $60 \%$ & $10.8(10.1)$ & $0.8-39$ & $52 \%$ \\
\hline$A B / A C$ deletion group & 15 & $86 \%$ & $8.9(4.2)$ & $2-15$ & $53 \%$ \\
\hline A-B deletion & 11 & $80 \%$ & $7.8(3.8)$ & $2-15$ & $55 \%$ \\
\hline $\mathrm{A}-\mathrm{C}$ deletion & 4 & $100 \%$ & $11.8(4.4)$ & $5-14$ & $50 \%$ \\
\hline $\mathrm{BD} / \mathrm{CD}$ deletion group & 18 & $69 \%$ & 11.1(10.7) & $1-38$ & $50 \%$ \\
\hline B-D deletion & 14 & $77 \%$ & $10.4(9.0)$ & $1-38$ & $43 \%$ \\
\hline C-D deletion & 4 & $33 \%$ & 13.6(16.9) & $0.8-36$ & $75 \%$ \\
\hline BD/CD duplication group & 10 & $13 \%$ & $14.5(15.2)$ & $1-39$ & $60 \%$ \\
\hline B-D duplication & 8 & $0 \%$ & 16.5(16.6) & $1-39$ & $63 \%$ \\
\hline C-D duplication & 2 & $50 \%$ & $6.5(2.0)$ & $5-7$ & $50 \%$ \\
\hline A-B duplication ${ }^{\mathrm{b}}$ & 1 & Unknown & 7.0 & - & $0 \%$ \\
\hline E-F duplication ${ }^{\mathrm{b}}$ & 2 & $0 \%$ & $6.6(2.8)$ & $4-8$ & $50 \%$ \\
\hline
\end{tabular}

Participant characteristics for all individuals with a nested deletion or duplication of 22q11.2, including three case studies with atypical nested duplications noted with superscript $b$

aPercentage of individuals with known inheritance

${ }^{b}$ Case studies not included in statistical analysis, medical chart review, or $A B / A C$ and $B D / C D$ group totals 
Three additional patients who carried very small and rare atypical duplications are included in this paper in a descriptive manner (in the case study sections), but are not combined with the other groups in tables, figures, or statistical analyses. One patient carried a very small duplication within LCR-A to LCR-B. The other two patients (who were related to three patients in the main LCR-B to D duplication group) carried a small duplication nested between LCR-E and LCR-F.

All 43 participants were included in the medical history chart review. Nine participants were excluded from the ASD and psychiatric symptom analyses $(n=34$; see Fig. 2) for two types of reasons: (1) ASD classification could not be determined ( $n=2$; see below) or (2) if they presented with another medical issue likely to affect brain development ( $n=2$ extreme prematurity and/or birth weight $<5$ th centile, $n=2$ with CEDNIK syndrome, $n=1$ with $16 \mathrm{p} 11.2$ deletion which is independently associated with ASD, $n=2$ history of hypoxic brain injury) [31-34]. Participant characteristics of the sample excluding these nine cases are described in Table 2. Please note that some ages differ from those in the medical record review (Table 1) because a review of updated records pertinent to ASD classification, when available, was conducted 3 years later to allow for infants to reach the age (3 years) at which ASD symptoms would be present.

Rates of autism were analyzed separately for individuals with nested deletions and duplications (see Table 3). Only one individual per family (the proband) was included to avoid confounding autism rates with risk factors shared by related individuals. In one family with BD duplication, we included an affected family member instead of the proband because the proband harbored a 16 p11.2 deletion. For deletions, 20 individuals were included after excluding five parents and younger siblings (2 B-D, 2 C-D, 1 A-B). For duplications, five individuals were included after excluding four parents and younger siblings (4 B-D). No individuals presented with nested duplications involving LCR-A to LCR-B or C.

\section{Comparison cohorts}

We compiled comparison questionnaire data from four cohorts. Detailed results of medical system chart review, neuropsychiatric questionnaires, ASD symptoms, and adaptive functioning of these four comparison groups have been published elsewhere [6]. Two cohorts were drawn from patients at the same clinic who had a

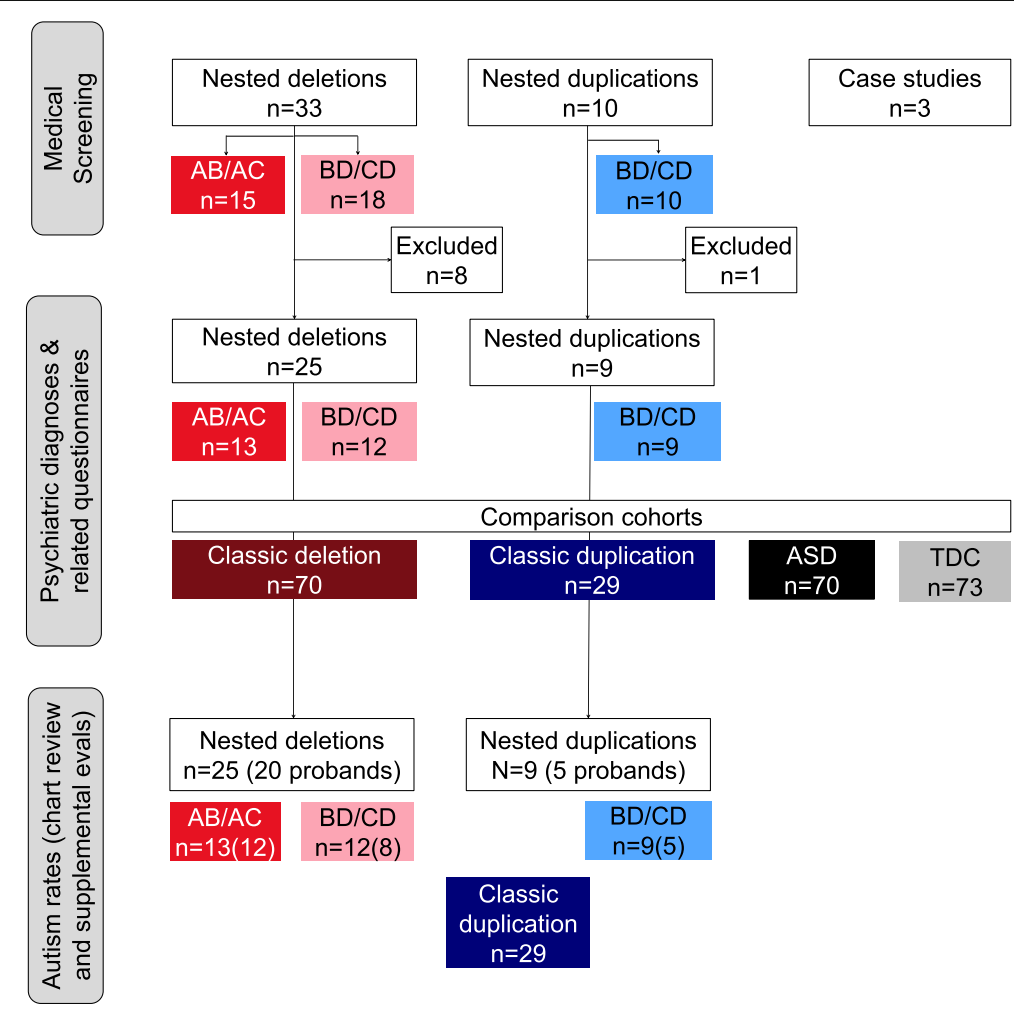

Fig. 2 Participant flow chart depicting the participants and comparisons included in each portion of the study. Group colors correspond to colors in Figs. 3 and 4 and Additional file 2. Abbreviations: AB/AC deletion or duplication spanning LCR-A to LCR-B or LCR-A to LCR-C, ASD non-syndromic autism spectrum disorder, $B D / C D$ deletion or duplication spanning LCR-B to LCR-D or LCR-C to LCR-D, classic deletion typical $22 q 11.2$ deletion syndrome involving LCR-A to D, classic duplication typical 22q11.2 duplication syndrome involving LCR-A to D, LCR low-Copy repeat region, TDC typically developing controls 
Table 2 Descriptive characteristics of participants included in psychiatric diagnosis rates

\begin{tabular}{|c|c|c|c|c|c|}
\hline Region & Number & $\%$ de novo ${ }^{a}$ & Age mean (sd) & Age range (years) & $\%$ male \\
\hline $\mathrm{AB} / \mathrm{AC}$ deletion group & 13 & $83 \%$ & $10(4.2)$ & $5-18$ & $54 \%$ \\
\hline A-B deletion & 10 & $78 \%$ & $8.9(3.6)$ & $5-15$ & $60 \%$ \\
\hline A-C deletion & 3 & $100 \%$ & $13.7(4.8)$ & $9-18$ & $33 \%$ \\
\hline $\mathrm{BD} / \mathrm{CD}$ deletion group & 12 & $50 \%$ & $14.2(12.7)$ & $3-42$ & $50 \%$ \\
\hline B-D deletion & 8 & $57 \%$ & $13.4(12.1)$ & $4-42$ & $38 \%$ \\
\hline C-D deletion & 4 & $33 \%$ & $15.9(15.5)$ & $3-37$ & $75 \%$ \\
\hline BD/CD duplication group & 9 & $14 \%$ & $16.9(14.7)$ & $5-39$ & $56 \%$ \\
\hline B-D duplication & 7 & $0 \%$ & $19.4(15.9)$ & $5-39$ & $57 \%$ \\
\hline C-D duplication & 2 & $50 \%$ & $8(4.1)$ & $5-11$ & $50 \%$ \\
\hline Total & 34 & $55 \%$ & $13.3(11.0)$ & $3-42$ & $53 \%$ \\
\hline
\end{tabular}

Participant characteristics for the subset of individuals with a nested deletion or duplication of 22 q11.2 included in description of psychiatric diagnosis rates ${ }^{\text {a }}$ Percentage of individuals with known inheritance

confirmed classic (LCR-A to LCR-D) 22q11.2 duplication $(n=29)$ or deletion $(n=70)$. A non-syndromic ASD cohort $(n=70)$ and typically developing control cohort $(n=73)$ were drawn from other studies of neurodevelopment at the CHOP Center for Autism Research. These four cohorts were age- and sex-matched to one another but were not as well matched to either of the small nested samples described above to allow for inclusion of all eligible individuals with a nested CNV.

Informed consent was obtained for all 22q11.2 participants, as well as for all participants in the comparison cohorts (Institutional Review Board protocols \#13101307, \#09-007275, \#07-005689, and \#10-007622).

\section{Procedures}

We collected data from record review, questionnaires administered remotely, and, for a subset, an autism-specific evaluation. Record review included the participant's electronic health record at $\mathrm{CHOP}$ whenever possible, as well as external medical and educational records (e.g., IEP evaluations) provided by families for individuals who did not receive routine medical care at our institution.

\section{Medical record and developmental history review}

Medical and developmental history was obtained from a questionnaire completed by the participant. A licensed pediatrician and medical geneticist (TLW) reviewed clinic notes, progress reports, radiology reports, laboratory reports, etc. in each participant's record to confirm key components reported by participants. Psychiatric and neurodevelopmental diagnostic history was documented in this process as it is routinely collected during clinical visits. Families were contacted by phone to resolve questions or discrepancies.

\section{ASD diagnostic classification}

Sources of diagnostic information Given that our hypotheses concerned rates of ASD, particular care was given to the ASD classification process. We assigned

Table 3 ASD rates among probands

\begin{tabular}{|c|c|c|c|c|c|c|}
\hline Region & Number & $n$ ASD (male) & $\%$ de novo ${ }^{a}$ & Age mean (sd) & Age range (years) & $\%$ male \\
\hline $\mathrm{AB} / \mathrm{AC}$ deletion group & 12 & $5(3)$ & $90 \%$ & $10.0(4.4)$ & $5-18$ & $58 \%$ \\
\hline A-B deletion & 9 & $4(3)$ & $90 \%$ & $8.7(3.7)$ & $5-15$ & $67 \%$ \\
\hline A-C deletion & 3 & $1(0)$ & $100 \%$ & $13.7(4.8)$ & $9-18$ & $33 \%$ \\
\hline $\mathrm{BD} / \mathrm{CD}$ deletion group & 8 & 0 & $60 \%$ & $10.5(4.8)$ & $5-18$ & $25 \%$ \\
\hline B-D deletion & 6 & 0 & $70 \%$ & $10.2(3.8)$ & $6-17$ & $17 \%$ \\
\hline C-D deletion & 2 & 0 & $0 \%$ & $11.6(9.1)$ & $5-18$ & $50 \%$ \\
\hline Classic AD duplication & 29 & $7(5)$ & $67 \%$ & $7.1(3.4)$ & $2-13$ & $75 \%$ \\
\hline BD/CD duplication group & 5 & $1(0)$ & $30 \%$ & $12.7(10.4)$ & $5-31$ & $60 \%$ \\
\hline B-D duplication & 3 & $1(0)$ & $0 \%$ & $15.8(13.1)$ & $7-31$ & $67 \%$ \\
\hline C-D duplication & 2 & 0 & $50 \%$ & $8.0(4.1)$ & 5-11 & $50 \%$ \\
\hline
\end{tabular}

Participant characteristics and autism diagnosis for all probands with a nested deletion of 22q11.2. Individuals harboring an AB or AC deletion presented with ASD at a rate of $41.6 \%$ ( 5 of 12 ). Case studies are excluded from this table

a Percentage of individuals with known inheritance 
diagnostic status after a thorough record review of clinical, research, and educational records provided by families and available in the CHOP electronic health record. Participants differed in the frequency with which they received documented $\mathrm{CHOP}$ care. Continuous longitudinal data from $\mathrm{CHOP}$ developmental pediatricians and psychiatrists existed for individuals who lived locally, whereas records of individuals who lived further away or moved sometimes contained only the initial "22q and You" clinic evaluation. Participants were also asked to provide external medical and educational records.

All families were invited for an in-person ASD evaluation using the Autism Diagnostic Observation Schedule (ADOS and ADOS-2), parent interview, and IQ testing to complete a DSM-5 (Diagnostic and Statistical Manual of Mental Illness, 5th edition) checklist [35, 36]. However, since many of our families lived far away, this proved infeasible for a large percentage of the cases. Families who could not complete an in-person evaluation were invited for an hour-long parent phone interview with a clinician asking follow-up questions to Social Communication Questionnaire, Lifetime (SCQ) responses to complete an accurate DSM-5 checklist [35, 36].

"ASD" group We assigned participants to the "ASD" group if there was documentation of an ASD diagnosis ( $n=5$ deletions, $n=1$ duplication). Five individuals had a diagnostic evaluation in their record; one did not, but had frequent references to the ASD diagnosis throughout the record. All participants scored above threshold (15) on the SCQ.

"No ASD" group We assigned "No ASD" ( $n=20$ deletions, $n=8$ duplications) if ASD had been considered but specifically ruled out ( $n=13$ deletions, $n=3$ duplications), or if there was no indication of ASD concerns in the available records $(n=7$ deletions, $n=5$ duplications). Two individuals (both LCR-B to D deletions) were excluded because a referral for an ASD evaluation had been recommended recently but not completed.

The absence of parental or professional concern about ASD is not routinely documented. Thus, we further investigated this group to determine whether there was a true absence of concern, or a lack of information. We studied parent/spouse report, provider report, behavioral descriptions, and referral history. The $22 \mathrm{q}$ clinic routinely refers to developmental behavioral pediatrics or psychiatry if parents indicate relevant concerns during intake, but parents without those concerns would not have had these appointments scheduled. The 22q clinic also routinely questions parents regarding developmental history and previous concern of psychiatric diagnoses from school or medical professionals, as this patient population is at high risk for psychosis and other psychiatric disorders. Any concerns and prior assessments are documented in detail. Therefore, we feel confident that families were routinely asked about developmental concerns and thus that a lack of referrals and text about concerns is a reasonably robust indicator of a lack of ASD concerns.

\section{Neuropsychiatric questionnaires}

We collected neuropsychiatric questionnaire data from participants under age 18. Questionnaires included a measure of adaptive functioning (Vineland Adaptive Behavior Scales-2nd Edition, "Vineland-II," completed for participants 0-18 years old [37]), a screener for psychiatric disorders based on DSM-IV checklists (Child and Adolescent Symptom Inventory-4R, "CASI-4R," completed for participants 5-18 years old depending on disorder [38]), and two measures of social behavior and autistic symptoms (Social Communication Questionnaire--Lifetime, "SCQ," completed for participants four and above [39], and the Social Responsiveness Scale or Social Responsiveness Scale, 2nd edition, "SRS-2" for participants 2.5-18 years old [40, 41]). Every questionnaire offers excellent psychometric properties, and all but the Social Communication Questionnaire provide standardized scores based on a large, representative norming sample. Please see Additional file 1 for characteristics of subsamples that completed each questionnaire.

\section{Analysis}

ASD rate To test our hypothesis that the LCR-A to LCR$B$ region might confer increased risk of ASD in 22q11.2 duplication and deletion syndromes, we compared ASD rates among individuals whose deletion affected the LCRA to LCR-B region ("AB/AC group": LCR-A to B, or LCR$A$ to $C)$ to individuals whose deletion did not affect the LCR-A to LCR-B region ("BD/CD group": LCR-B to D, and LCR-C to D). Thus, our first analysis compared the $\mathrm{AB} / \mathrm{AC}$ group to the $\mathrm{BD} / \mathrm{CD}$ group for deletions only. In a second, more conservative analysis, we compared only individuals with deletions of LCR-A to B to those with LCR$\mathrm{B}$ to $\mathrm{D}$ (excluding cases with deleted LCR-A to C or LCR$\mathrm{C}$ to $\mathrm{D})$ to match the groups on approximate size and number of genes in the deletion.

Rates were compared using a one-tailed Fisher's exact test to account for cells with $n<5$. An odds ratio (OR) cannot be computed when certain cells contain 0 observations; in these cases, we present $95 \%$ confidence intervals and $p$ values from Fisher's exact test and effect sizes as chi-square statistics.

Our sample included no individuals with nested duplications involving LCR-A to $\mathrm{B}$ (i.e., no " $\mathrm{AB} / \mathrm{AC}$ " group for duplications). Thus, we compared the $\mathrm{BD} / \mathrm{CD}$ duplication group to individuals with the classic LCR-A to D duplication, which does involve LCR-A to B. These results are provided for descriptive purposes only due to the sample size 
of the nested duplications, which although is one of the largest reported, remains quite small .

Psychiatric symptoms (standardized questionnaires) In our dimensional analysis of psychiatric symptoms using questionnaire data, we analyzed raw scores on the SCQ, age-normed scores on the Vineland-II and SRS-2, and symptom composite scores on the CASI-4R. For deletions, we compared individuals in the "BD/CD" group to the $A B / A C$ group . For duplications, we compared individual in the $\mathrm{BD} / \mathrm{CD}$ group to the comparison cohort of classic duplications because our sample included no $\mathrm{AB} / \mathrm{AC}$ duplications. We also compare the $\mathrm{AB} / \mathrm{AC}$ deletion group to the classic deletion group as this information might prove directly useful clinically. Our interpretations focus on the size of the effect and its confidence interval, as opposed to inferential statistics, to avoid making overly strong statements based on a small sample, as suggested by many recent position papers, e.g., Button et al. [42] and Cumming et al. [43]. We present the effect sizes for each analysis and make our data available upon request so that the data generated here can be leveraged in any future meta-analyses to test our hypothesis directly.

Medical and psychiatric diagnoses We present rates of psychiatric and medical comorbidities by nested region separately for individuals who did and did not receive recommended screening. All analyses are descriptive and for characterization purposes only. Statistical significance was not tested due to small sample sizes within each nested region.

\section{Results}

Higher rates of ASD when LCR-A to $B$ involved

We observed a trend toward a higher rate of ASD among probands with deletions in the $\mathrm{AB} / \mathrm{AC}$ group $(41.7 \%$, or 5 in 12 individuals with LCR-A to B, or LCR-A to C) compared to the $\mathrm{BD} / \mathrm{CD}$ group $(0 \%$, or 0 in 8 individuals with LCR-B to D, or LCR-C to D; $\mathrm{X}=4.4, p=0.051$, CI 0.99 , Inf; see Table 3 ). In a more conservative analysis that matched groups on approximate size of deleted region, we continued to observe similar rates of ASD within each group (44.4\%, or 4 of 9 individuals with deletions of LCR$\mathrm{A}$ to $\mathrm{B}$, and $0 \%$, or 0 in 6 individuals with deletions of LCR-B to D; $\mathrm{X}=3.64, p=0.092$, CI 0.702, Inf). The rate of ASD did not change meaningfully when related individuals were included to increase sample size; the increased sample size provided more statistical power and revealed significant results $(n=25 ; 38.5 \%$ rate in $\mathrm{AB} / \mathrm{AC}$ group, $0 \%$ in $\mathrm{BD} / \mathrm{CD}$ group; $\mathrm{X}=5.77, p=0.024$, CI 1.39, Inf). Thus, the LCR-A to $B$ region may confer increased risk of ASD diagnosis, but a larger sample without related individuals is needed to confim.

Among duplications, individuals with the classic and $\mathrm{BD} /$ CD duplications showed similar rates of ASD (24.1\% rate or 7 of 29 in classic group, $20 \%$ rate or 1 of 5 in $\mathrm{BD} / \mathrm{CD}$; $\mathrm{OR}=0.79, p=0.764$, CI 0.03, Inf). Results did not change meaningfully when related individuals were included to increase sample size (21.4\% rate in classic group, $11.1 \%$ rate or 1 of 9 in $\mathrm{BD} / \mathrm{CD}$; OR $=0.40, p=0.65$, CI 0.02, Inf), but this analysis in particular would benefit from a larger sample.

Our categorical analysis was supported by quantitative reports of autistic symptoms in the SRS-2 and SCQ (see Fig. 3). A subset of each group (BD/CD deletions, $A B / A C$ deletions, $\mathrm{BD} / \mathrm{CD}$ duplications, classic duplications, classic
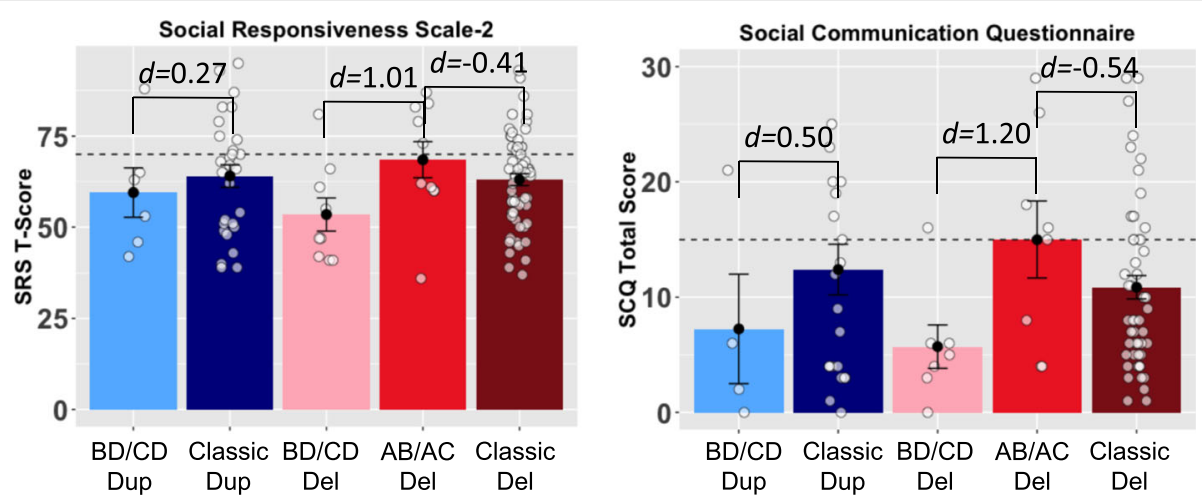

Fig. 3 Individuals with deleted LCR-A to B show higher levels of autistic symptoms. This figure depicts group means, standard errors, and the effect size of differences on two quantitative measures of autistic symptoms, the Social Responsiveness Scale-2 and the Social Communication Scale, Lifetime. Each point depicts one individual. Dashed lines indicate the threshold above which an individual is considered to screen positive for autism and warrant further evaluation. The groups with involvement of LCR-A to B show higher levels of social impairment, with large effect sizes for deletions and small to medium effect sizes for duplications. Effect sizes are not significant due to small samples (see Table 4). The AB/AC deletion group includes five individuals diagnosed with autism; the BD/CD deletion group includes zero. Abbreviations: $A B / A C$ deletion spanning LCR-A to LCR-B or LCR-A to LCR-C; $B D / C D$ deletion or duplication spanning LCR-B to LCR-D or LCR-C to LCR-D; $d$ Cohen's $d$ effect size; del deletion; dup duplication; LCR low-Copy repeat region; SCQ Social Communication Questionnaire, Lifetime; SRS Social Responsiveness Scale 

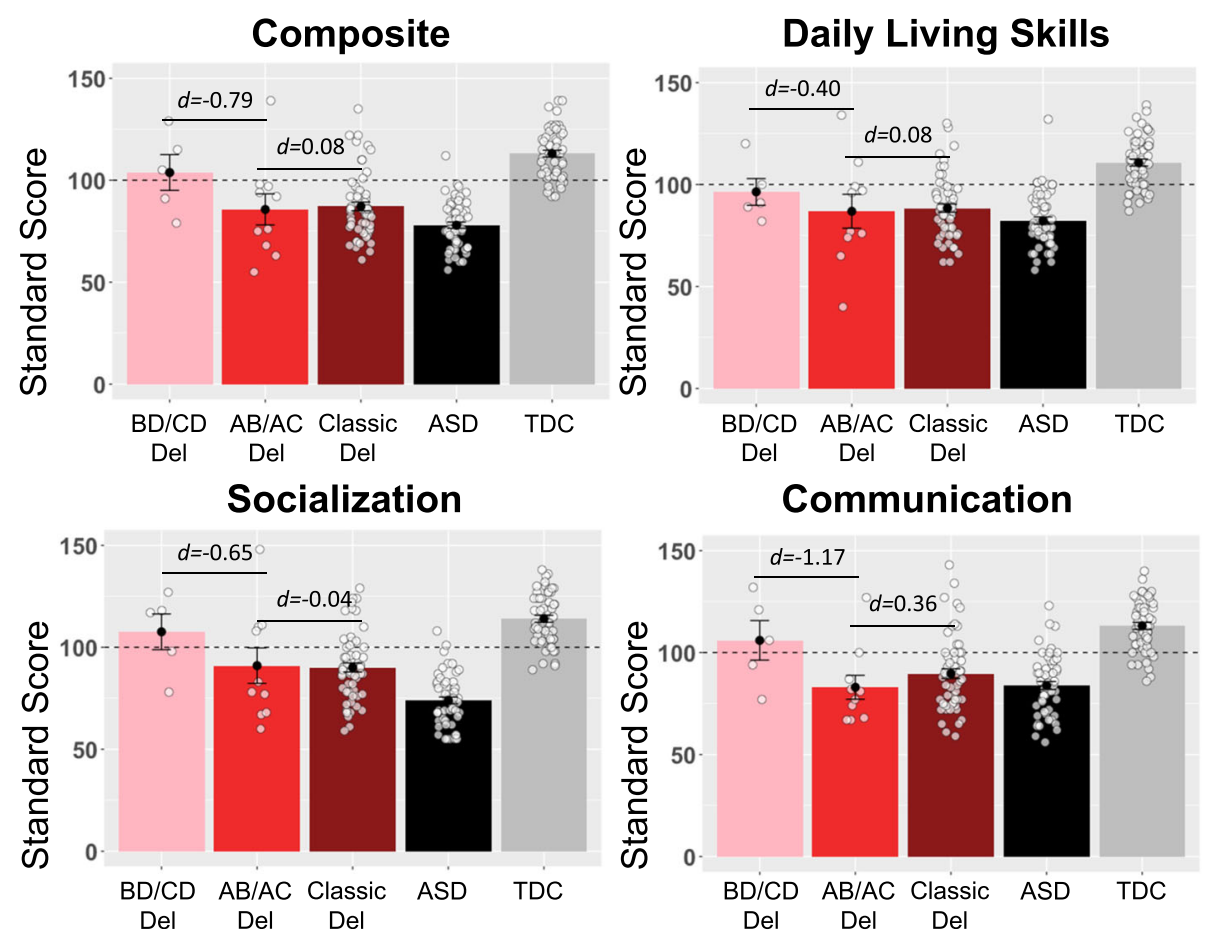

Fig. 4 Individuals with deleted LCR-A to B show modestly lower levels of adaptive functioning on the Vineland-II Adaptive Behavior Scales. This figure depicts group means, standard errors, and the effect size of differences on the Vineland-II, a measure of adaptive behavior. Each point depicts one individual). Higher scores on the Vineland-II indicate higher levels of functioning across the three domains and composite score, and standard scores are age-normed such that 100 (represented by the dashed line) indicates average. The AB/AC deletion group shows more impairment than the BD/ $C D$ nested deletions that do not involve $L C R-A$ to $B$, with medium to large effect sizes; the $A B / A C$ group also shows similar levels of impairment to the classic deletion group (deletion spanning LCR-A to D), with very small effect sizes. Abbreviations: $A B / A C$ deletion spanning LCR-A to LCR-B or LCR-A to LCR-C, ASD individuals with non-syndromic autism spectrum disorder, BD/CD deletion spanning LCR-B to LCR-D or LCR-C to LCR-D, $d$ Cohen's $d$ effect size, del deletion, LCR low copy repeat region, TDC typically developing controls

deletions) completed the SCQ, including both individuals with and without ASD diagnoses. For deletions, the BD/CD group showed less autistic symptoms than the $A B / C D$ group with large effect sizes ( $d$ 's of 1.01 and 1.20). For duplications, the difference was small-to-medium ( $d$ 's of 0.27 and 0.50 ) between the $\mathrm{BD} / \mathrm{CD}$ group and the classic group. No effects reached statistical significance (see Table 4).

\section{Moderately lower adaptive and social functioning when $A B$ region involved}

We computed effect sizes for differences in autistic symptoms, psychiatric symptoms, and adaptive behavior skills (see Figs. 3 and 4, Table 4, Additional file 2). For duplications, the differences were usually small between the $\mathrm{BD} / \mathrm{CD}$ group and the classic duplication group (see Table 4, "Classic Duplication" rows). For deletions, the $\mathrm{BD} / \mathrm{CD}$ group showed less impairment than the $\mathrm{AB} / \mathrm{AC}$ group across most measures with medium or large effect sizes that did not reach statistical significance. We also calculated effect sizes for group differences between the $\mathrm{AB} / \mathrm{AC}$ deletions and classic $\mathrm{AD}$ deletion groups and observed small or medium differences (see Table 4, "Classic Deletion" rows). We observed negligible differences between these two groups on most adaptive functioning scales. The classic deletion group showed slightly lower levels of autistic symptoms compared to the $A B /$ AC group--small to medium effect sizes on the SRS-2 and SCQ--that were not statistically significant.

\section{Increased rates of psychiatric disorders}

In individuals with nested duplications or deletions, we observed elevated rates compared to population means in nearly every psychiatric disorder reported, including ADHD, OCD, ODD and related behaviors, depression, language disorders, global developmental delay, and intellectual disability. See Table 5 for observed rates of disorders by type of nested deletion or duplication.

\section{Higher rates of medical comorbidities}

We documented presence or absence of having received an appropriate screening test, and whether or not an 
Table 4 Group means and effect sizes of group differences on neuropsychiatric questionnaires

\begin{tabular}{|c|c|c|c|c|}
\hline & Number & Mean (SD) & $d$ & $\begin{array}{l}95 \% \text { confidence } \\
\text { interval }\end{array}$ \\
\hline SRS-2 T score & & $50(10)$ & & \\
\hline $\mathrm{BD} / \mathrm{CD}$ deletion & 9 & $53.4(13.6)$ & & \\
\hline $\mathrm{AB} / \mathrm{AC}$ deletion & 10 & $68.5(15.7)$ & 1.01 & $(-0.07,2.11)$ \\
\hline Classic deletion & 61 & $63.0(12.6)$ & -0.41 & $(-1.11,0.27)$ \\
\hline BD/CD duplication & 6 & $59.5(16.6)$ & & \\
\hline Classic duplication & 28 & $64.0(16.3)$ & 0.27 & $(-0.67,1.22)$ \\
\hline SCQ raw total & & Cutoff 15 & & \\
\hline $\mathrm{BD} / \mathrm{CD}$ deletion & 7 & $5.7(4.9)$ & & \\
\hline $\mathrm{AB} / \mathrm{AC}$ deletion & 8 & $15.0(9.3)$ & 1.20 & $(-0.11,2.52)$ \\
\hline Classic deletion & 52 & $10.8(7.3)$ & -0.54 & $(-1.32,0.23)$ \\
\hline BD/CD duplication & 4 & $7.2(9.5)$ & & \\
\hline Classic duplication & 22 & $12.4(10.3)$ & 0.50 & $(-0.67,1.68)$ \\
\hline Vineland composite & & $100(15)$ & & \\
\hline $\mathrm{BD} / \mathrm{CD}$ deletion & 5 & $103.8(19.6)$ & & \\
\hline $\mathrm{AB} / \mathrm{AC}$ deletion & 10 & $85.7(24.0)$ & -0.79 & $(-2.11,0.52)$ \\
\hline Classic deletion & 57 & $87.1(15.9)$ & 0.08 & $(-0.61,0.78)$ \\
\hline BD/CD duplication & 6 & $92.6(18.7)$ & & \\
\hline Classic duplication & 27 & $89.4(19.4)$ & -0.16 & $(-1.11,0.78)$ \\
\hline Vineland communication & & 100(15) & & \\
\hline $\mathrm{BD} / \mathrm{CD}$ deletion & 5 & $106.0(21.7)$ & & \\
\hline $\mathrm{AB} / \mathrm{AC}$ deletion & 10 & $83.0(18.5)$ & -1.17 & $(-2.55,0.20)$ \\
\hline Classic deletion & 58 & $89.7(18.1)$ & 0.36 & $(-0.32,1.06)$ \\
\hline BD/CD duplication & 6 & $92.1(20.2)$ & & \\
\hline Classic duplication & 27 & $91.2(18.5)$ & -0.04 & $(-0.99,0.90)$ \\
\hline Vineland daily living & & $100(15)$ & & \\
\hline $\mathrm{BD} / \mathrm{CD}$ deletion & 5 & $96.4(14.6)$ & & \\
\hline $\mathrm{AB} / \mathrm{AC}$ deletion & 10 & $86.9(26.2)$ & -0.40 & $(-1.69,0.87)$ \\
\hline Classic deletion & 57 & $88.4(15.1)$ & 0.08 & $(-0.60,0.78)$ \\
\hline BD/CD duplication & 6 & $91.8(23.0)$ & & \\
\hline Classic duplication & 28 & $93.6(21.2)$ & 0.08 & $(-0.86,1.02)$ \\
\hline Vineland socialization & & $100(15)$ & & \\
\hline $\mathrm{BD} / \mathrm{CD}$ deletion & 5 & $107.6(19.6)$ & & \\
\hline $\mathrm{AB} / \mathrm{AC}$ deletion & 10 & $91.0(27.4)$ & -0.65 & $(-1.96,0.65)$ \\
\hline Classic deletion & 57 & $90.1(16.5)$ & -0.04 & $(-0.74,0.64)$ \\
\hline BD/CD duplication & 6 & $98.1(18.3)$ & & \\
\hline Classic duplication & 27 & $90.8(21.2)$ & -0.35 & $(-1.30,0.60)$ \\
\hline \multicolumn{5}{|l|}{ CASI ADHD } \\
\hline $\mathrm{BD} / \mathrm{CD}$ deletion & 2 & $2.5(1.3)$ & & \\
\hline $\mathrm{AB} / \mathrm{AC}$ deletion & 7 & $3.4(1.4)$ & 0.63 & $(-1.56,2.83)$ \\
\hline Classic deletion & 43 & $2.7(1.2)$ & -0.52 & $(-1.36,0.32)$ \\
\hline BD/CD duplication & 4 & $2.7(2.1)$ & & \\
\hline Classic duplication & 20 & $2.8(1.3)$ & 0.09 & $(-1.09,1.27)$ \\
\hline
\end{tabular}

Table 4 Group means and effect sizes of group differences on neuropsychiatric questionnaires (Continued)

\begin{tabular}{|c|c|c|c|c|}
\hline & Number & Mean (SD) & $d$ & $\begin{array}{l}95 \% \text { confidence } \\
\text { interval }\end{array}$ \\
\hline BD/CD deletion & 2 & $1.3(0.7)$ & & \\
\hline $\mathrm{AB} / \mathrm{AC}$ deletion & 7 & $1.9(1.1)$ & 0.49 & $(-1.68,2.67)$ \\
\hline Classic deletion & 43 & $2.3(1.3)$ & 0.30 & $(-0.53,1.14)$ \\
\hline BD/CD duplication & 4 & $1.9(1.5)$ & & \\
\hline Classic duplication & 20 & $1.8(1.6)$ & -0.05 & $(-1.24,1.12)$ \\
\hline \multicolumn{5}{|l|}{ CASI ASD } \\
\hline BD/CD deletion & 1 & $\mathrm{n} / \mathrm{a}$ & & \\
\hline $\mathrm{AB} / \mathrm{AC}$ deletion & 4 & $1.0(1.0)$ & $\mathrm{n} / \mathrm{a}$ & n/a \\
\hline Classic deletion & 37 & $0.5(0.4)$ & -0.83 & $(-1.94,0.27)$ \\
\hline BD/CD duplication & 4 & $0.6(0.4)$ & & \\
\hline Classic duplication & 16 & $0.8(0.8)$ & 0.26 & $(-0.98,1.50)$ \\
\hline \multicolumn{5}{|l|}{ CASI Schizoaffective } \\
\hline BD/CD deletion & 1 & $\mathrm{n} / \mathrm{a}$ & & \\
\hline $\mathrm{AB} / \mathrm{AC}$ deletion & 3 & $0.5(0.5)$ & $\mathrm{n} / \mathrm{a}$ & $\mathrm{n} / \mathrm{a}$ \\
\hline Classic deletion & 6 & $0.5(0.2)$ & -0.09 & $(-0.78,0.61)$ \\
\hline BD/CD duplication & 0 & $\mathrm{n} / \mathrm{a}$ & & \\
\hline Classic duplication & 4 & $0.5(0.2)$ & $\mathrm{n} / \mathrm{a}$ & $\mathrm{n} / \mathrm{a}$ \\
\hline \multicolumn{5}{|l|}{ CASI behav. regulation } \\
\hline BD/CD deletion & 2 & $0.9(0.4)$ & & \\
\hline $\mathrm{AB} / \mathrm{AC}$ deletion & 7 & $0.9(0.6)$ & 0.06 & $(-2.08,2.21)$ \\
\hline Classic deletion & 43 & $1.0(0.6)$ & 0.16 & $(-0.67,1.00)$ \\
\hline BD/CD duplication & 4 & $0.3(0.3)$ & & \\
\hline Classic duplication & 20 & $1.0(0.8)$ & 0.78 & $(-0.42,2.00)$ \\
\hline \multicolumn{5}{|l|}{ CASI Depression } \\
\hline BD/CD deletion & 2 & $0.4(0)$ & & \\
\hline $\mathrm{AB} / \mathrm{AC}$ deletion & 7 & $0.6(0.7)$ & 0.33 & $(-1.82,2.50)$ \\
\hline Classic deletion & 43 & $0.5(0.7)$ & -0.15 & $(-0.99,0.67)$ \\
\hline BD/CD duplication & 4 & $0.2(0.4)$ & & \\
\hline Classic duplication & 20 & $0.4(0.4)$ & 0.48 & $(-0.70,1.68)$ \\
\hline
\end{tabular}

Group means on neuropsychiatric questionnaires. We show $95 \%$ confidence intervals of effect sizes as Cohen's $d$, which can be interpreted as follows: 0.2 as small, 0.5 as medium, and 0.8 as large [54]. Means and standard deviations for each group are presented, as well as the mean and SD for each measure to aid in interpretation. We derived SRS T-scores using the updated SRS-2 norms for all participants, regardless of the version the participant completed. We averaged CASI$4 \mathrm{R}$ raw item scores on similar subscales into composites instead of using $\mathrm{T}$-scores because we encountered a strong ceiling effect when using CASI-4R T-scores because CASI-4R norms collapse all high raw scores into a T-score of 70 . Thus, population-normed means and standard deviations are not available for comparison for these averaged composites. For example, all items from the dysthymia subscale and major depression subscales were averaged into a "Depression" composite, after accounting for the number of items in each subscale so that both scales were weighted equally in the composite. The composites are interpreted as " 3 " indicating that on average, the parent endorsed symptoms in the domain as occurring "very often," 2 as "often," 1 as "sometimes," and 0 as "never"

Vineland Vineland Adaptive Behavior Scales, 2nd Edition; CASI Child and Adolescent Symptom Inventory-4R; SCQ Social Communication Questionnaire; SRS-2 Social Responsiveness Scale, 2nd Edition; $n / a$ not applicable 
Table 5 Psychiatric disorder rates from parent and adult self-report and chart review

\begin{tabular}{|c|c|c|c|c|c|c|c|c|c|c|c|}
\hline & Total $(n)$ & No eval & Had eval & GDD & Lang Dx & $\mathrm{ADHD}$ & ID & ODD & OCD & Anxiety & MDD \\
\hline $\mathrm{AB} / \mathrm{AC}$ deletion & 13 & $7.7 \%$ & $92.3 \%$ & $30.8 \%$ & $0.0 \%$ & $53.8 \%$ & $0.0 \%$ & $7.7 \%$ & $23.1 \%$ & $23.1 \%$ & $0.0 \%$ \\
\hline A-B deletion & 10 & $10 \%$ & $90 \%$ & $30 \%$ & $0 \%$ & $50 \%$ & $0 \%$ & $10 \%$ & $20 \%$ & $20 \%$ & $0 \%$ \\
\hline$<3$ yrs & 1 & 1 & 0 & 0 & 0 & 0 & 0 & 0 & 0 & 0 & 0 \\
\hline $3-14$ yrs & 8 & 0 & 8 & 2 & 0 & 4 & 0 & 0 & 1 & 1 & 0 \\
\hline $15+y r s$ & 1 & 0 & 1 & 1 & 0 & 1 & 0 & 1 & 1 & 1 & 0 \\
\hline A-C deletion & 3 & $0 \%$ & $100 \%$ & $33.3 \%$ & $0 \%$ & $66.7 \%$ & $0 \%$ & $0 \%$ & $33.3 \%$ & $33.3 \%$ & $0 \%$ \\
\hline $3-14$ yrs & 1 & 0 & 1 & 1 & 0 & 0 & 0 & 0 & 0 & 0 & 0 \\
\hline $15+y r s$ & 2 & 0 & 2 & 0 & 0 & 2 & 0 & 0 & 1 & 1 & 0 \\
\hline $\mathrm{BD} / \mathrm{CD}$ deletion & 12 & $33.3 \%$ & $66.7 \%$ & $16.7 \%$ & $8.3 \%$ & $8.3 \%$ & $25 \%$ & $8.3 \%$ & $25 \%$ & $25 \%$ & $0 \%$ \\
\hline B-D deletion & 8 & $12.5 \%$ & $87.5 \%$ & $37.5 \%$ & $0 \%$ & $12.5 \%$ & $12.5 \%$ & $0 \%$ & $0 \%$ & $25 \%$ & $25 \%$ \\
\hline$<3 \mathrm{yrs}$ & 1 & 1 & 0 & 0 & 0 & 0 & 0 & 0 & 0 & 0 & 0 \\
\hline $3-14$ yrs & 5 & 0 & 5 & 3 & 0 & 1 & 1 & 0 & 0 & 0 & 0 \\
\hline $15+y r s$ & 2 & 0 & 2 & 0 & 0 & 0 & 0 & 0 & 0 & 2 & 2 \\
\hline C-D deletion & 4 & $75 \%$ & $25 \%$ & $0 \%$ & $0 \%$ & $25 \%$ & $0 \%$ & $25 \%$ & $25 \%$ & $25 \%$ & $25 \%$ \\
\hline$<3 \mathrm{yrs}$ & 2 & 2 & 0 & 0 & 0 & 0 & 0 & 0 & 0 & 0 & 0 \\
\hline $15+y r s$ & 2 & 1 & 1 & 0 & 0 & 1 & 0 & 1 & 1 & 1 & 1 \\
\hline BD/CD duplication & 9 & $33.3 \%$ & $66.6 \%$ & $22.2 \%$ & $0 \%$ & $0 \%$ & $44.4 \%$ & $0 \%$ & $11.1 \%$ & $11.1 \%$ & $11.1 \%$ \\
\hline B-D duplication & 7 & $42.9 \%$ & $57.1 \%$ & $28.6 \%$ & $14.3 \%$ & $28.6 \%$ & $0 \%$ & $0 \%$ & $0 \%$ & $14.3 \%$ & $14.3 \%$ \\
\hline$<3$ yrs & 1 & 1 & 0 & 0 & 0 & 0 & 0 & 0 & 0 & 0 & 0 \\
\hline $3-14$ yrs & 3 & 0 & 3 & 2 & 1 & 1 & 0 & 0 & 0 & 1 & 0 \\
\hline $15+y r s$ & 3 & 2 & 1 & 0 & 0 & 1 & 0 & 0 & 0 & 0 & 1 \\
\hline C-D duplication & 2 & $0 \%$ & $100 \%$ & $100 \%$ & $0 \%$ & $0 \%$ & $0 \%$ & $0 \%$ & $0 \%$ & $0 \%$ & $0 \%$ \\
\hline $3-14$ yrs & 2 & 0 & 2 & 2 & 0 & 0 & 0 & 0 & 0 & 0 & 0 \\
\hline Total sample & 34 & $23.5 \%$ & $76.5 \%$ & $32.3 \%$ & $2.9 \%$ & $32.3 \%$ & $2.9 \%$ & $5.9 \%$ & $11.8 \%$ & $20.6 \%$ & $11.8 \%$ \\
\hline
\end{tabular}

We observed elevated rates of psychiatric diagnoses among individuals with nested duplications or deletions relative to population base rates using parent- and self-report data confirmed in medical records. Among the full sample, $77 \%$ had received a psychiatric evaluation. The most commonly reported diagnoses in our sample included ADHD and Global Developmental Delay (GDD), which may reflect the sample's skew toward younger ages (see Table 2 for sample characteristics). We present rates for group totals, and we present $n$ 's for age bins based roughly on when documentation of diagnosis would be expected (i.e., GDD and language disorders are frequently diagnosed before age $3, \mathrm{ADHD}$ and ID are usually diagnosed in childhood after age 3 , and depression and anxiety frequently onset during adolescence or adulthood) to facilitate interpretation of overall group rates because rates for disorders that frequently appear in adolescence (e.g., anxiety and depression) are likely underestimates

Abbreviations: Eval evaluation; $A D H D$ attention deficit/hyperactivity disorder; ID intellectual disability; $O C D$ obsessive compulsive disorder; GDD Global Developmental Delay; Lang Dx language disorder, receptive or expressive; ODD oppositional defiant disorder; MDD major depressive disorder; Del deletion; Dup duplication; yrs years

abnormality was identified, in individuals with nested deletions and duplications between LCR-A and D (see Table 6). In order to calculate conservative estimates for the prevalence of each medical comorbidity in each group, we report both the percentage of screened individuals and the percentage of total individuals.

\section{Case study 1}

Isolating specific genes: an individual with ASD and tiny duplication involving RANBP1 and COMT, not TBX1

One individual in our sample came to attention of clinical geneticists due to autism spectrum disorder and was found to have a small, $300 \mathrm{~kb}$ microduplication within the LCR-A to $\mathrm{B}$ region that included RANBP1 and COMT but not
TBX1. Detailed clinical evaluation and all recommended medical screening for individuals with 22q11.2-related disorders revealed none of the medical issues or dysmorphic features characteristic of the syndrome. However, the individual met diagnostic criteria for ASD, anxiety, and ADHD after evaluation by a neurodevelopmental pediatrician and standardized neuropsychiatric evaluation. The inheritance of this microduplication is unknown because parental testing was not possible. To our knowledge, no relatives carry an autism diagnosis but none have received formal evaluation. The individual's SNP array showed no other pathogenic variants. This individual was not included in group analyses because the duplication did not encompass the full LCR-A to B region. 


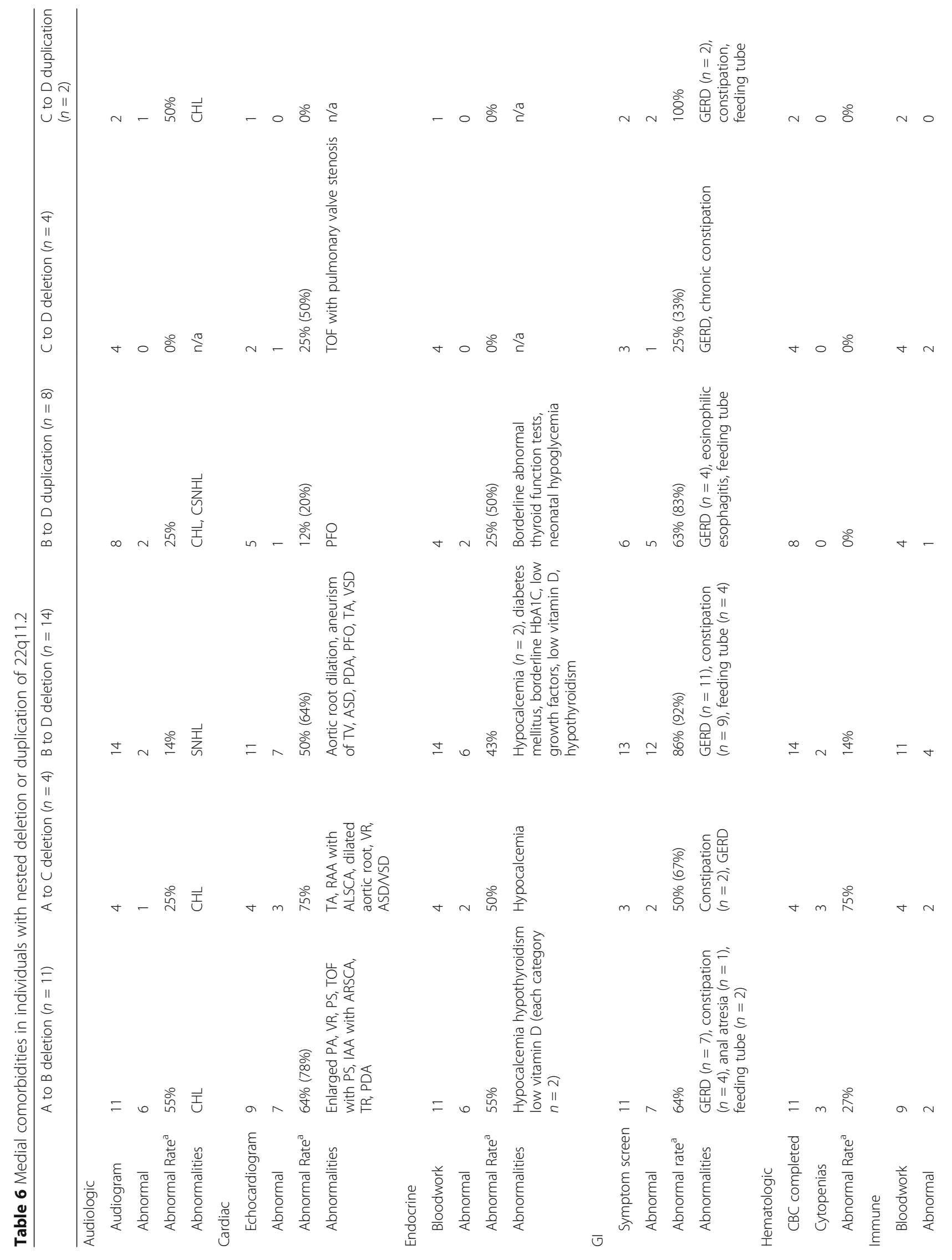




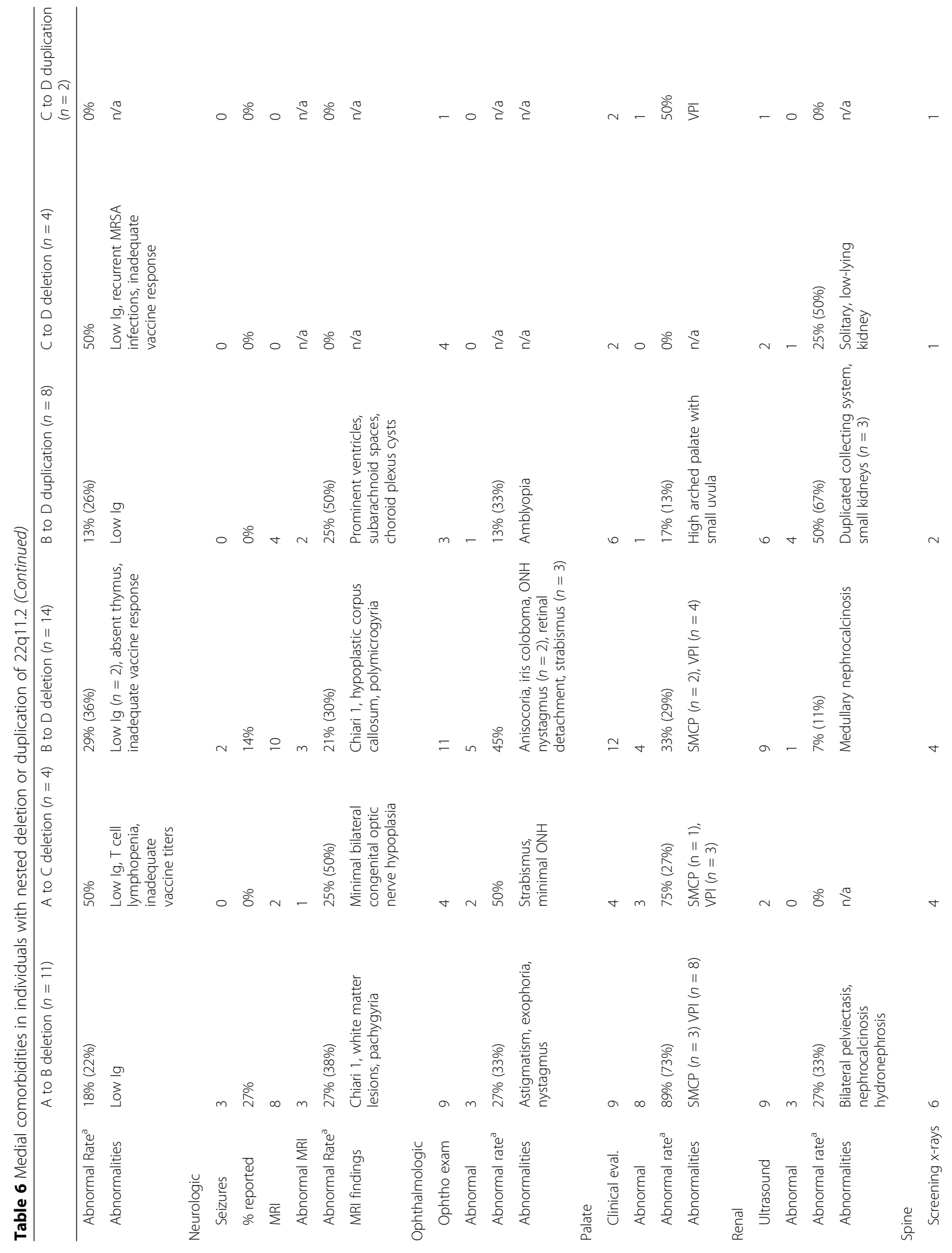




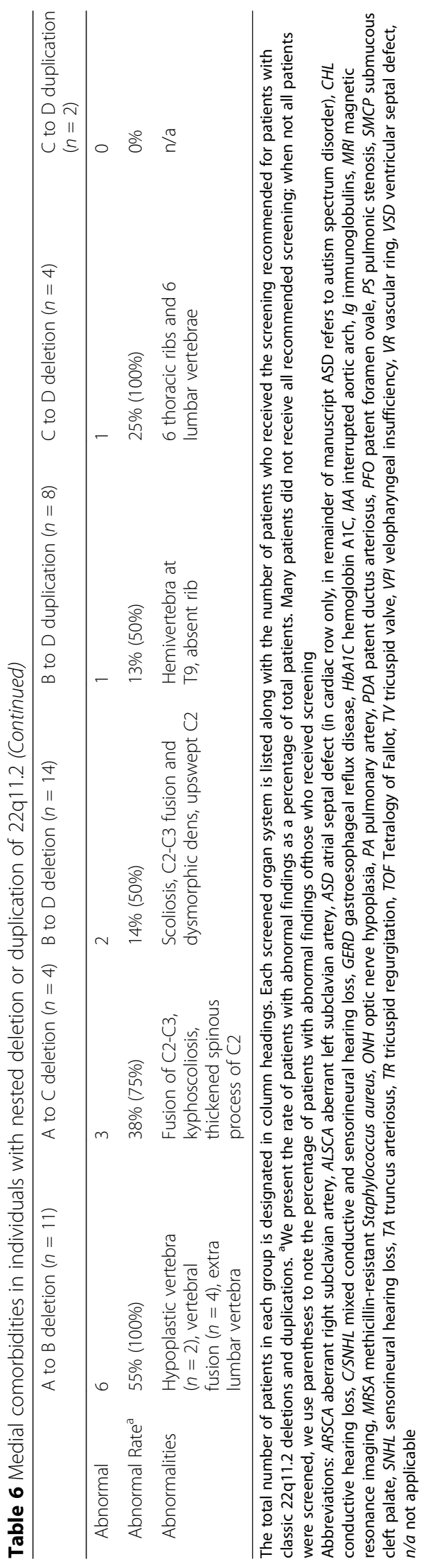




\section{Case study 2}

The role of background genetics: a family with $L C R-B$ to $D$ duplication and distal E-F duplication and autism and face processing deficits

The only individual in our analyses with autism in the $\mathrm{BD} / \mathrm{CD}$ group carried a duplication of LCR-B to D. She had one sibling with the same LCR-B to D duplication and two siblings with a duplication of TOP $3 B$ (in a small region between LCR-E and F). One of the siblings with the TOP3B duplication had a history of an autism diagnosis but did not currently present with significant autism symptoms. Furthermore, the proband and the sibling with LCR-B to D duplication both showed decreased face processing abilities on the Benton Facial Recognition Test (mildly impaired in the proband, clinically impaired in the sibling). Face processing difficulties have not been reported in 22q syndromes before, and we do not posit that they are central to the syndromes, but rather that the family history of possible ASD and the genetic complexity of the family raises the question that other genetic factors may have contributed to the proband's autism. Future studies of autism in nested 22q11.2 should evaluate family members for ASD and prosopagnosia, and evaluate probands for phenotypes seen in other family members, to better understand the contribution of background genetics.

\section{Discussion}

To our knowledge, this study includes the largest group of individuals with nested deletions and duplications of $22 \mathrm{q} 11.2$ to be compared prospectively to classic deletions and duplications with standardized measures. These data suggest that individuals with deletion of the LCR-A to B region may have a higher rate of ASD (39-44\%) than those without involvement (0\%); the pattern was not replicated for duplications. Taken in conjunction with case study 1 , these findings are consistent with our hypothesis that LCR-A to B may confer risk for ASD in 22q11.2 related disorders. However, we offer this evidence as preliminary support that requires further exploration with additional samples.

It is notable that the nested deletions of all individuals with ASD involved LCR-A to B and that we observed negligible differences between this group and the classic deletion spanning LCR-A to D in adaptive functioning. These results suggest that LCR-A to B could be contributing to the autistic phenotype in individuals with classic 22q11.2DS, as well as to decreased adaptive functioning. It is also notable that we observed no duplications of LCR-A to B or LCR-A to C in our full sample of 43 individuals, although such individuals are mentioned in much larger studies [44]. Thus, it remains to be tested in larger samples whether these individuals are as likely to present with ASD as those with the classic A-D duplication.

\section{Implications for medical screening}

Prior studies have suggested that individuals with nested deletions have similar types of medical problems to those with classic deletions and should receive similar clinical treatment. The medical chart review of our patients supported this hypothesis. It also suggested that our patients are representative of other previously reported patients with nested deletions with regard to the frequency and types of medical problems. It is notable that there appeared to be fewer medical problems in individuals with LCR-C to D. However, this region is much smaller, encompassing fewer genes than the other regions. In size and total number of genes, LCR-A to LCR-B and LCR-B to LCR-D are roughly equivalent, and the rates of medical comorbidities are similar. We also observed higher rates of some medical comorbidities in several of the nested groups as compared to individuals with full LCR-A to LCR-D deletions (e.g., cervical spine anomalies in $100 \%$ of screened individuals with LCR-A to LCR-B deletion), but our sample sizes are too small to determine if this is due to chance or truly represents a higher risk subgroup. We were somewhat surprised to find that many patients had not completed portions of the recommended medical screening for individuals with 22q11.2-related disorders. It is unclear if this is due to a perception by providers that individuals with nested deletions do not need as aggressive screening as those with full deletions or duplications. Overall, we observed rates of each of the medical comorbidities in the LCR-A to LCR-B and LCR-B to LCR-D subgroups that are comparable to rates in individuals with full LCR-A to LCR-D deletions or duplications. Although the rate of medical problems appears lower in the LCR-C to LCR-D deletion and duplication groups, the sample sizes are extremely small, and therefore, no strong conclusions can be made about the validity of an altered screening protocol for these patients.

\section{RANBP1 as a potential ASD candidate gene}

The LCR-A to B region associated with ASD risk in our sample involves approximately 25 genes, including COMT, $P R O D H$, and TBX1. Prior research implicates the interaction of low activity COMT and $P R O D H$ alleles in ASD risk $[28,29]$. Other genes in the region may also confer ASD risk, and indeed, the risk could be additive. We propose another possible candidate gene, Ran-binding protein 1 (RANBP1), which could not be examined given our study design with multiple candidate genes in the LCRA to $B$ region, warranting further investigations. We base this speculation on five circumstantial pieces of evidence.

First, we cite the involvement of RANBP1 in the metabotropic glutamate receptor (mGluR) gene network [44], which is disrupted in two other syndromic forms of ASD, fragile $\mathrm{X}$ syndrome and tuberous sclerosis complex [45]. Second, we previously observed a 10 -fold increase 
in ASD rate among individuals with 22q11.2DS with a "second hit" in an mGluR network gene compared to individuals without a "second hit" (5 affected in 25 individuals with 22q11.2 compared to 1 in 50) [30]. Third, two teratogens associated with increased rates of ASD--valproate and thalidomide--both decrease expression of RANBP1 [46, 47, 48]. Fourth, the important link between $R A N B P 1$ and expression in human brains was demonstrated by Meechan et al. [49], who showed higher RANBP1 expression in developing fetal brains compared to adult brains during a peak in neurogenesis. Finally, several studies in the 22q11.2 animal literature highlight $R A N B P 1$ as important for neural development in 22q11.2 (e.g., [49-51]). Taken together, these disparate pieces of literature converge on a role of RANBP1 in brain development, and potentially in ASD. Like other genes and gene families recently associated with ASD, RANBP1 serves a general function within the cell (metabolizing GTP and regulating material transport to the nucleus [52]). RANBP1 has not been identified previously as an ASD candidate gene in large ASD studies; of the approximately 25 genes in the 22q11.2 LCR-A to LCR-B region, previous genome-wide association studies or whole exome sequencing studies have identified $P R O D H$ as a candidate gene with suggestive evidence and TBX1 and GNB1L as candidate genes with minimal evidence at this time (SFARI gene database https:/gene.sfari.org/database/human-gene/). It is not yet clear whether genes in this region modify ASD risk in the general population or in the context of $22 \mathrm{q} 11.2$ syndromes alone.

\section{Insights from two case studies involving $T B X 1$ and RANBP1}

Individuals with very small nested duplications and deletions offer a unique method of studying the associations between isolated regions or genes and individual features of the 22q11.2DS phenotype. In the present study, we could not tease apart the contributions of individual genes to portions of the phenotype, as the LCR-A to B region includes approximately 25 genes. Here, we contrast two case studies, case study 1 and a prior case study by Weisfeld-Adams and colleages [53], each with a very small duplication including either $T B X 1$ or $R A N B P 1$, but not both, to provide some insight into the possible relative contributions of $T B X 1$ and $R A N B P 1$ to the phenotype in a descriptive fashion. Weisfeld-Adams et al. described a patient and sibling with duplication of six genes including TBX1 but not RANBP1. This proband showed complex medical problems, but neither the 19-month-old proband nor the 3-year-old sibling showed any symptoms of autism or neurodevelopmental delay besides mild motor delay. (Although no concern for ASD was noted at 19 months of age, we caution against over-interpretation because ASD can be missed in toddlers when symptoms are not severe. However, by 19 months of age most children with 22q11.2DS show significant delays, little speech, and aloof social behavior, so the lack of delay suggests social development was on course.) In contrast, in case study 1 , we described an individual with microduplication involving RANBP1 but not TBX1 who had ASD but no medical comorbidities. Both our patient, who had a purely psychiatric phenotype and duplication that does involve RANBP1, and the case presented by Weisfeld-Adams et al.--a purely medical phenotype that does not involve RANBP1--provide preliminary suggestive evidence that $R A N B P 1$, not $T B X 1$, specifically might confer risk for ASD and other psychiatric diagnoses. Both microduplications include $C O M T$ and exclude $P R O D H$, so we cannot speculate about the roles of these genes based on case studies.

\section{Limitations}

The two primary limitations of our study lie in the phenotyping and the sample size. This single-site study relied primarily on questionnaires and chart review, supplemented by in-person evaluation when feasible for the family. Thus, the phenotyping, while accurate, could be improved with systematic prospective evaluations. Our sample size was small, owing to the rarity of individuals with nested duplication or deletions in the 22q11.2 region. Our study would benefit from replication with a multi-site study that combines clinics around the world to improve statistical power.

Another limitation includes the unknown role of background genetics. We were unable to account for other contributors to ASD risk, such as common variants or known pathogenic variants occurring outside 22q11.2 that would be identified with whole-exome sequencing, not clinical genetic testing with MLPA and SNP arrays. However, this risk is likely to affect all groups equally. Furthermore, we believe this unknown potential risk is likely to be small compared to the known, larger ASD risk of carrying 22q11.2DS or DupS.

Future directions might involve whole-exome sequencing of 22q11.2 samples to identify other factors that contribute to ASD risk. Such a study should include an analysis leveraging the sequencing of $P R O D H, C O M T$, RANBP1, and TBX1 in individuals with nested 22q11.2 deletions and duplications to isolate the influence of these mutations on the ASD phenotype.

\section{Conclusions}

We present data on medical and psychiatric issues in 44 individuals with nested duplications and deletions within the LCR-A to D region, along with two additional siblings with tiny duplication of $T O P 3 B$, the largest cohort of this type to be studied prospectively. We found 
increased rate of ASD among individuals with deleted LCR-A to B, compared to individuals whose nested deletions did not involve that region. We tentatively speculate that $R A N B P 1$ could provide a potential mechanistic explanation for increased rates of ASD based on this finding, our reported case study, environmental ASD risk factors that also alter RANBP1 expression, RANBP1's role in the mGluR network, and the role of the mGluR network in other syndromic forms of ASD. We also conclude from our observation of the full spectrum of medical issues in each group that at this time, there is insufficient evidence to limit medical screening in individuals with nested duplications or deletions within the 22q11.2 region.

\section{Additional files}

Additional file 1: This file depicts participant characteristics for each questionnaire. (DOCX $26 \mathrm{~kb})$

Additional file 2: This file depicts higher parent-reported psychiatric symptoms among individuals with nested or classic 22q11.2 duplication or deletions compared to typically developing controls. (DOCX $266 \mathrm{~kb}$ )

\section{Abbreviations \\ 22q11.2DS: 22q11.2 deletion syndrome; 22q11.2DupS: 22q11.2 duplication syndrome; ADHD: Attention-deficit/hyperactivity disorder; ADI-R: Autism Diagnostic Interview-Revised; ADOS: Autism Diagnostic Observation Schedule; ASD: Autism spectrum disorder; CASI-4R: Child and Adolescent Symptom Inventory-4R; CHOP: Children's Hospital of Philadelphia; CNV: Copy number variation; COMT: Catechol-O-methyltransferase; DSM: Diagnostic and Statistical Manual of Mental Disorders; FXS: Fragile X syndrome; LCR: Low-copy repeat; mGluR: Metabotropic glutamate receptor; MLPA: Multiplex ligation probe amplification; Ran: Ras-related nuclear protein; RANBP1: Ran-binding protein 1; SCQ: Social Communication Questionnaire; SNP: Single nucleotide polymorphism; SRS: Social Responsiveness Scale; TDC: Typically developing children; Vineland-II: Vineland Adaptive Behavior Scales-II}

\section{Acknowledgements}

The authors wish to thank Katie Lowe, Karen Liao, Joy Payton, and all of the families who graciously offered their time to participate in the study, especially the families of patients highlighted in the case studies.

\section{Funding}

This study was funded by grants from the Simons Foundation Autism Research Initiative (275687), the National Institutes of Child and Human Development (U54 HD86984, R01 HD055741), the Pennsylvania Department of Health (SAP \#4100042728, SAP \#4100047863), the Robert Wood Johnson Foundation (\#66727), and the National Institute of Mental Health (RC1MH08879, 5R01MH107431-02). This material is based upon work supported by the National Science Foundation Graduate Research Fellowship Program under grant no. DGE-1321851.

\section{Availability of data and materials}

The datasets used and/or analyzed during the current study are available from the corresponding author on reasonable request. The dataset is not being published in a repository for the sole reason that the combination of sex, age, and diagnostic information about a very rare genetic condition could be used to identify individuals who participated in this study.

\section{Authors' contributions}

TW conceived of the study, assisted in identification of participants, performed medical chart review, and assisted in data interpretation and manuscript preparation. CCC was involved in project conception and design, performed psychological assessments on participants, performed psychological record review, provided all statistical analysis, interpreted results, and drafted the manuscript. AZ and JB recruited participants, administered questionnaires, collected data, and contributed to data analysis and editing of the manuscript. JSM was involved in experimental design, supervised and performed psychological assessments on participants, and edited the manuscript. AdM contributed to project design, performed assessments on participants, performed psychological record review, and edited the manuscript. JCC contributed to participant recruitment and edited the manuscript. BEY contributed to assessments of participants, consulted on statistical analysis approach, and edited the manuscript. LDP contributed to subject recruitment, administration of questionnaires, collected data, and reviewed the manuscript. BE, EZ, and DMM were involved in conception of the project, subject recruitment, confirmed cytogenetic diagnosis, and reviewed the manuscript. RTS contributed to all phases of the project including design, implementation, synthesis of results, analysis, and review of the manuscript. All authors approved the manuscript.

Ethics approval and consent to participate

Informed consent for participation was obtained for all participants; these documents and procedures had been approved by the Children's Hospital of Philadelphia Insitutional Review Board (protocols \#13-101307, \#13-101307, \#09-007275, \#07-005689, \#10-007622).

\section{Consent for publication}

Both families with individual data presented gave written consent for publication.

\section{Competing interests}

The authors declare that they have no competing interests.

\section{Publisher's Note}

Springer Nature remains neutral with regard to jurisdictional claims in published maps and institutional affiliations.

\section{Author details}

${ }^{1}$ Center for Autism Research, The Children's Hospital of Philadelphia, 2716 South Street, Philadelphia, PA 19104, USA. ²Department of Psychology, University of Pennsylvania, 3720 Walnut Street, Philadelphia, PA 19104, USA. ${ }^{3}$ Department of Pediatrics, Seattle Children's Hospital, 4800 Sand Point Way NE, Seattle, WA 98105, USA. ${ }^{4}$ Vanderbilt Brain Institute, Vanderbilt University School of Medicine, 465 21st Avenue South, Nashville, TN 37232, USA. ${ }^{5}$ Department of Psychiatry, University of Pennsylvania, 3400 Civic Center Boulevard, Philadelphia, PA 19104, USA. ${ }^{6}$ Department of Behavioral and Social Science, University of the Sciences, 600 South 43rd Street, Philadelphia, PA 19104, USA. 'Department of Pediatrics, The Children's Hospital of Philadelphia, 3400 Civic Center Boulevard, Philadelphia, PA 19104, USA. ${ }^{8}$ Department of Pediatrics, University of Utah, Salt Lake City, UT 84108, USA.

Received: 1 July 2017 Accepted: 27 September 2017 Published online: 27 October 2017

\section{References}

1. McDonald-McGinn DM, Emanuel BS, Zackai EH. 22q11.2 Deletion Syndrome. In: Pagon RA, Adam MP, Ardinger HH, Wallace SE, Amemiya A, Bean LJ, et al., editors. GeneReviews $\left({ }^{\circledR}\right)$. Seattle (WA): University of Washington, Seattle; 1999. http://www.ncbi.nlm.nih.gov/books/NBK1523/.

2. Grati FR, Molina Gomes D, Ferreira JCPB, Dupont C, Alesi V, Gouas L, et al. Prevalence of recurrent pathogenic microdeletions and microduplications in over 9500 pregnancies. Prenat Diagn. 2015;35:801-9.

3. McDonald-McGinn DM, Kirschner R, Goldmuntz E, Sullivan K, Eicher P, Gerdes M, et al. The Philadelphia Story: the 22q11. 2 deletion: report on 250 patients. Genet Couns Geneva Switz. 1999;10:11-24.

4. Wentzel C, Fernström M, Öhrner Y, Annerén G, Thuresson A-C. Clinical variability of the 22q11. 2 duplication syndrome. Eur J Med Genet. 2008;51:501-10.

5. Kobrynski LJ, Sullivan KE. Velocardiofacial syndrome, DiGeorge syndrome: the chromosome 22q11.2 deletion syndromes. Lancet. 2007;370:1443-52.

6. Wenger TL, Miller JS, DePolo LM, de Marchena AB, Clements CC, Emanuel BS, et al. 22q11.2 duplication syndrome: elevated rate of autism spectrum disorder and need for medical screening. Mol Autism. 2016;7 doi:10.1186/ s13229-016-0090-z.

7. Schneider M, Debbané M, Bassett AS, Chow EWC, Fung WLA, van den Bree $M$, et al. Psychiatric disorders from childhood to adulthood in $22 q 11.2$ deletion syndrome: results from the International Consortium on Brain and Behavior in 22q11.2 Deletion Syndrome. Am J Psychiatry. 2014;171:627-39. 
8. Hoeffding LK, Trabjerg BB, Olsen L, Mazin W, Sparsø T, Vangkilde A, et al. Risk of psychiatric disorders among individuals with the 22q11.2 deletion or duplication: a danish nationwide, register-based study. JAMA Psychiatry. 2017;74:282-90.

9. Rees E, Kirov G, Sanders A, Walters JR, Chambert KD, Shi J, et al. Evidence that duplications of 22q11. 2 protect against schizophrenia. Mol Psychiatry. 2014;19:37.

10. Vorstman JAS, Morcus MEJ, Duijff SN, Klaassen PWJ, Heineman-de Boer JA, Beemer FA, et al. The 22q11.2 deletion in children: high rate of autistic disorders and early onset of psychotic symptoms. J Am Acad Child Adolesc Psychiatry. 2006;45:1104-13.

11. Ousley O, Evans AN, Fernandez-Carriba S, Smearman EL, Rockers K, Morrier MJ, et al. Examining the overlap between autism spectrum disorder and 22q11.2 deletion syndrome. Int J Mol Sci. 2017;18:1071.

12. Angkustsiri K, Goodlin-Jones B, Deprey L, Brahmbhatt K, Harris S, Simon TJ. Social impairments in chromosome 22q11.2 deletion syndrome (22q11.2DS): autism spectrum disorder or a different endophenotype? J Autism Dev Disord. 2014;44:739-46.

13. Michaelovsky E, Frisch A, Carmel M, Patya M, Zarchi O, Green T, et al. Genotype-phenotype correlation in 22q11. 2 deletion syndrome. BMC Med Genet. 2012;13:122.

14. Bengoa-Alonso A, Artigas-Lopez M, Moreno-Igoa M, Cattalli C, HernandezCharro B, Ramos-Arroyo MA. Delineation of a recognizable phenotype for the recurrent LCR22-C to D/E atypical 22q11.2 deletion. Am J Med Genet A. 2016;170:1485-94.

15. Guris DL, Fantes J, David T, Druker BJ, Imamoto A. Mice lacking the homologue of the human 22q11. 2 gene CRKL phenocopy neurocristopathies of DiGeorge syndrome. Nat Genet. 2001;27:293.

16. Lindsay EA, Vitelli F, Su H, Morishima $M$, et al. Tbx 1 haploinsufficiency in the DiGeorge syndrome region causes aortic arch defects in mice. Nature. 2001;410:97.

17. Guo T, McDonald-McGinn D, Blonska A, Shanske A, Bassett AS, Chow E, et al. Genotype and cardiovascular phenotype correlations with TBX1 in 1,022 velo-cardio-facial/digeorge/22q11. 2 deletion syndrome patients. Hum Mutat. 2011:32:1278-89.

18. Jerome LA, Papaioannou VE. DiGeorge syndrome phenotype in mice mutant for the T-box gene, Tbx1. Nat Genet. 2001;27:286-91.

19. Herman SB, Guo T, McGinn DMM, Blonska A, Shanske AL, Bassett AS, et al. Overt cleft palate phenotype and TBX1 genotype correlations in velocardio-facial/DiGeorge/22q11. 2 deletion syndrome patients. Am J Med Genet A. 2012:158:2781-7.

20. Raux G, Bumsel E, Hecketsweiler B, van Amelsvoort T, Zinkstok J, ManouvrierHanu S, et al. Involvement of hyperprolinemia in cognitive and psychiatric features of the 22q11 deletion syndrome. Hum Mol Genet. 2006;16:83-91.

21. Jacquet H, Demily C, Houy E, Hecketsweiler B, Bou J, Raux G, et al. Hyperprolinemia is a risk factor for schizoaffective disorder. Mol Psychiatry. 2005;10:479

22. Gothelf D, Eliez S, Thompson T, Hinard C, Penniman L, Feinstein C, et al. COMT genotype predicts longitudinal cognitive decline and psychosis in 22q11. 2 deletion syndrome. Nat Neurosci. 2005;8:1500.

23. Vorstman JA, Turetsky BI, Sijmens-Morcus ME, de Sain MG, Dorland B, Sprong $\mathrm{M}$, et al. Proline affects brain function in 22q11DS children with the low activity COMT158 allele. Neuropsychopharmacology. 2009;34:739.

24. Bassett AS, Caluseriu O, Weksberg R, Young DA, Chow EW. Catechol-Omethyl transferase and expression of schizophrenia in 73 adults with 22911 deletion syndrome. Biol Psychiatry. 2007;61:1135-40.

25. Baker K, Baldeweg T, Sivagnanasundaram S, Scambler P, Skuse D. COMT Val 108/158 Met modifies mismatch negativity and cognitive function in 22q11 deletion syndrome. Biol Psychiatry. 2005;58:23-31.

26. Murphy $K C$, Jones LA, Owen MJ. High rates of schizophrenia in adults with velo-cardio-facial syndrome. Arch Gen Psychiatry. 1999;56:940-5.

27. Bassett AS, Chow EW. Schizophrenia and 22q11. 2 deletion syndrome. Curr Psychiatry Rep. 2008;10:148

28. Radoeva PD, Coman IL, Salazar CA, Gentile KL, Higgins AM, Middleton FA, et al. Association between autism spectrum disorder in individuals with velocardiofacial (22q11.2 deletion) syndrome and PRODH and COMT genotypes. Psychiatr Genet. 2014;24:269-72.

29. Hidding E, Swaab H, Sonneville LMJ, Engeland H, Vorstman J a S. The role of COMT and plasma proline in the variable penetrance of autistic spectrum symptoms in 22q11.2 deletion syndrome. Clin Genet. 2016;90:420-7.

30. Wenger TL, Kao C, McDonald-McGinn DM, Zackai EH, Bailey A, Schultz RT, et al. The role of mGluR copy number variation in genetic and environmental forms of syndromic autism spectrum disorder. Sci Rep. 2016;6:19372.
31. Snyder LG, D'Angelo D, Chen Q, Bernier R, Goin-Kochel RP, Wallace AS, et al. Autism spectrum disorder, developmental and psychiatric features in 16p11. 2 duplication. J Autism Dev Disord. 2016;46:2734-48.

32. Fuchs-Telem D, Stewart H, Rapaport D, Nousbeck J, Gat A, Gini M, et al. CEDNIK syndrome results from loss-of-function mutations in SNAP29. Br J Dermatol. 2011;164:610-6.

33. D'Angelo D, Lebon S, Chen Q, Martin-Brevet S, Snyder LG, Hippolyte L, et al. Defining the effect of the 16p11.2 duplication on cognition, behavior, and medical comorbidities. JAMA Psychiatry. 2016;73:20-30.

34. Dudova I, Markova D, Kasparova M, Zemankova J, Beranova S, Urbanek T, et al. Comparison of three screening tests for autism in preterm children with birth weights less than 1,500 grams. Neuropsychiatr Dis Treat. 2014;10:2201.

35. Lord C, Rutter M, DiLavore PC, Risi S. Autism diagnostic observation schedule (ADOS). Los Angeles, CA: Western Psychological Services; 1999.

36. American Psychiatric Association. Diagnostic and Statistical Manual. 5th ed. Arlington, VA: American Psychiatric Publishing; 2013.

37. Sparrow SS, Cicchetti DV, Balla DA. Vineland adaptive behavior scales, 2nd edition (Vineland II): survey interview form/caregiver rating form. Livonia, MN: Pearson Assessments; 2005.

38. Gadow KD, Sprafkin J. Child and adolescent symptom inventory-4R. Stony Brook, NY: Checkmate Plus; 2005.

39. Rutter M, Bailey A, Lord C. The Social Communication Questionnaire. Los Angeles, CA: Western Psychological Services; 2003.

40. Constantino JN, Gruber CP. Social Responsiveness Scale (SRS). Torrance, CA: Western Psychological Services; 2012.

41. Constantino JN, Gruber CP. Social Responsiveness Scale, 2nd edition (SRS-2). Los Angeles, CA: Western Psychological Services; 2012.

42. Button KS, loannidis JPA, Mokrysz C, Nosek BA, Flint J, Robinson ESJ, et al. Power failure: why small sample size undermines the reliability of neuroscience. Nat Rev Neurosci. 2013;14:365-76.

43. Cumming G. The new statistics: why and how. Psychol Sci. 2014;25:7-29.

44. Hadley D, Wu Z, Kao C, Kini A, Mohamed-Hadley A, Thomas K, et al. The impact of the metabotropic glutamate receptor and other gene family interaction networks on autism. Nat Commun. 2014;5 doi:10.1038/ ncomms5074.

45. Auerbach BD, Osterweil EK, Bear MF. Mutations causing syndromic autism define an axis of synaptic pathophysiology. Nature. 2011;480:63-8.

46. Jennifer L Ingram, Stacey M Peckham, Barbara Tisdale, Patricia M Rodier, (2000) Prenatal exposure of rats to valproic acid reproduces the cerebellar anomalies associated with autism. Neurotoxicology and Teratology 22(3): 319-324.

47. Jakob Christensen, Therese Koops Grønborg, Merete Juul Sørensen, Diana Schendel, Erik Thorlund Parner, Lars Henning Pedersen, Mogens Vestergaard, (2013) Prenatal Valproate Exposure and Risk of Autism Spectrum Disorders and Childhood Autism. JAMA 309 (16):1696.

48. Kesavan Meganathan, Smita Jagtap, Vilas Wagh, Johannes Winkler, John Antonydas Gaspar, Diana Hildebrand, Maria Trusch, Karola Lehmann, Jürgen Hescheler, Hartmut Schlüter, Agapios Sachinidis, Yao Liang Tang, (2012) Identification of Thalidomide-Specific Transcriptomics and Proteomics Signatures during Differentiation of Human Embryonic Stem Cells. PLoS ONE 7 (8):e44228.

49. Meechan DW, Maynard TM, Wu Y, Gopalakrishna D, Lieberman JA, LaMantia A-S. Gene dosage in the developing and adult brain in a mouse model of 22q11 deletion syndrome. Mol Cell Neurosci. 2006;33:412-28.

50. Paronett EM, Meechan DW, Karpinski BA, LaMantia A-S, Maynard TM. Ranbp1, deleted in DiGeorge/22q11.2 deletion syndrome, is a microcephaly gene that selectively disrupts layer $2 / 3$ cortical projection neuron generation. Cereb Cortex. 2015;25:3977-93.

51. Meechan DW, Tucker ES, Maynard TM, LaMantia A-S. Diminished dosage of $22 q 11$ genes disrupts neurogenesis and cortical development in a mouse model of 22q11 deletion/DiGeorge syndrome. Proc Natl Acad Sci. 2009;106:16434-45.

52. Zhang MS, Arnaoutov A, Dasso M. RanBP1 governs spindle assembly by defining mitotic Ran-GTP production. Dev Cell. 2014;31:393-404.

53. Weisfeld-Adams JD, Edelmann L, Gadi IK, Mehta L. Phenotypic heterogeneity in a family with a small atypical microduplication of chromosome 22q11.2 involving TBX1. Eur J Med Genet. 2012;55:732-6.

54. Cohen J. The effect size. Stat Power Anal Behav Sci. 1988:2:284-8. 